\title{
Development of Polycrystalline Silicon Based Photonic Crystal Membrane for Mid-Infrared Applications
}

\author{
Chong Pei Ho, Prakash Pitchappa, Piotr Kropelnicki, Jian Wang, Yuandong Gu, \\ and Chengkuo Lee, Member, IEEE
}

\begin{abstract}
Free-standing polycrystalline silicon ( $\mathrm{Si}$ ) based photonic crystal (PhC) membranes with etched circular and square holes are developed to display high reflectivity in the mid-infrared (MIR) region. Greater than $90 \%$ reflection was measured in the MIR wavelengths around $3.58 \mu \mathrm{m}$. By using square air holes in the $\mathrm{PhC}$ membrane, the mechanical strength of the polycrystalline $\mathrm{Si}$ membrane can be enhanced as square air holes have a lower filling factor of $36 \%$ of air holes, compared to $49 \%$ in circular air holes while keeping the reflectance around $3.45 \mu \mathrm{m}$ more than $90 \%$. Such Si PhC membranes offer opportunities for specific applications like filters. To illustrate the feasibility of such devices, simulation works are done by configuring two Si PhC membranes to create a FabryPerot interferometer operating in MIR region. The filtered peak shows a full width half maximum of $0.08 \mathrm{~nm}$ which corresponds to a quality factor of around 43800 , thus demonstrating the possibility of high-resolution applications such as gas sensing and hyperspectral imaging.
\end{abstract}

Index Terms-Fabry-Perot, optical properties, photonic crystals, thin films.

\section{INTRODUCTION}

$\mathbf{O}$ PTICAL reflectors play an important role in the realization of many optoelectronic devices and photonic elements such as mirrors, sensors and interferometers. Such feature of high reflectivity has been demonstrated by various methods as research in the field gained pace. Sub-wavelength metal gratings have been shown to produce broadband reflection over a large bandwidth but are hindered due to its high intrinsic absorption losses [1]-[6]. Multi-stacked dielectric Bragg reflector

Manuscript received October 11, 2013; revised November 29, 2013; accepted December 2, 2013. This work was supported support by SERC Grant 1021650084 from A*STAR, Singapore, and MOE2012-T2-2-154 (Monolithic Integrated Si/AIN Nanophotonics Platform for Optical NEMS and OEICs) under WBS No. R-263-000-A59-112

C. P. Ho and P. Pitchappa are with the Department of Electrical and Computer Engineering, National University of Singapore, Singapore 117576, and also with the Institute of Microelectronics, Agency for Science, Technology and Research, Singapore 117685 (e-mail: elehcp@nus.edu.sg; elepp@ nus.edu.sg).

P Kropelnicki, J. Wang, and Y. Gu, are with the Institute of Microelectronics, Agency for Science, Technology and Research, Singapore 117685 (e-mail: kropelnickip@ime.a-star.edu.sg; wangj@ime.a-star.edu.sg; guyd@ ime.a-star.edu.sg).

C. Lee is with the Department of Electrical and Computer Engineering, National University of Singapore, Singapore 117576 (e-mail: elelc@ nus.edu.sg).

Color versions of one or more of the figures in this paper are available online at http://ieeexplore.ieee.org.

Digital Object Identifier 10.1109/JSTQE.2013.2294463 is another common method used to produce highly reflective surface [7]-[10]. Alternating thin film of contrasting refractive index is deposited to produce a multi-layered structure which has displayed very low loss. However, the choice of the materials for the individual layers is limited due to the stringent requirement of the refractive index. In addition, the thickness of the layers has little tolerances and this poses a significant challenge in fabrication. These problems are more compounded when realizing such multi-layered structures to work in the mid-infrared (MIR), far-infrared (FIR) and THz regions as the thicker layers have more residual stress.

Recently, silicon $(\mathrm{Si})$ photonic crystal $(\mathrm{PhC})$ has attracted much attention due to its small size and exceptional optical performance. In particular, two dimensional (2-D) $\mathrm{PhC}$ has been shown to display Fano resonance [11]-[14] which is a characteristic of extremely high reflection. With properly designed parameters, high reflection can practically be achieved across different frequencies region. 2- $\mathrm{D} \mathrm{PhC}$ based single layer membrane devices have been reported by various groups for their high reflectivity in the optical communication applications, i.e., relevant wavelength of about $1550 \mathrm{~nm}$ [15]-[20], as well as in the MIR and FIR wavelength region [11], [21]-[24]. These reports show the potential of $\mathrm{PhC}$ membranes in practical applications.

There is however a lack of the investigation in using polycrystalline $\mathrm{Si}$ for the formation for such $\mathrm{PhC}$ membrane. This is mainly due to the challenge of the stress present in the membrane which usually causes buckling [25], [26] and even cracking. An attempt to achieve a free-standing polycrystalline $\mathrm{Si} \mathrm{PhC}$ membrane was done by Kim et al. in the NIR range [27]. In order to alleviate the stress present in the polycrystalline Si membrane, the authors used tensile stressed silicon nitride for stress compensation. However, such techniques require good matching between the stress in the $\mathrm{PhC}$ membrane and silicon nitride, which is difficult in typical deposition processes.

One major application of highly reflective surfaces is in the formation of Fabry-Perot interferometer (FPI) where two highly reflective surfaces are placed parallel to each other. The gap between the mirrors is designed to be $n \lambda / 2$, where $n$ is an integer and $\lambda$ is the desired filtered wavelength, in order to achieve constructive interference of the desired wavelength. The gap between the two highly reflective surfaces can be tuned by incorporating microelectromechanical systems (MEMS) technology. Such tunable filters are extremely important in applications like gas sensing [28], [29] and hyperspectral imaging [30], [31]. 
(a)

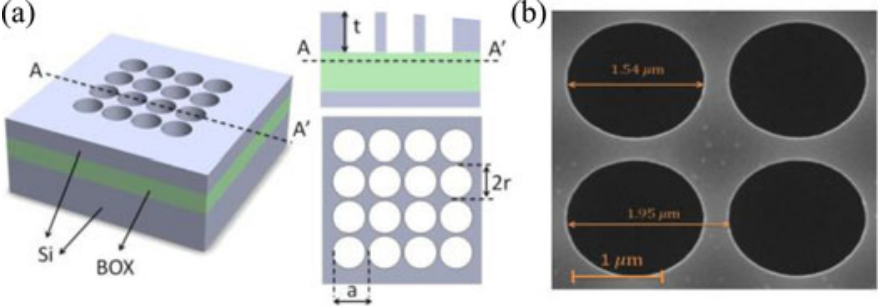

Fig. 1. (a) Schematic of the structure with circle air holes and (b) SEM image of the fabricated device.

Many attempts in recent works use multi-layered structure to achieve high reflectivity [29], [30], [32]-[34]. However, multilayered structures are typically heavy and require high actuation voltage to achieve tunability. In addition, when working at longer wavelengths, the thickness of the layers has to be increased and this complicates fabrication. Suh et al. introduced the idea of using 2-D PhC as the reflective mirror and proved theoretically the high performance of such FPI [35].

In this paper, we report the design, fabrication and characterization of ultra-compact polycrystalline $\mathrm{Si}$ based 2-D PhC membranes working in the MIR range. Two designs of the PhC membranes with circular and square air holes were investigated and characterized to show greater than $90 \%$ reflectivity in the MIR wavelength range around $3.45 \mu \mathrm{m}$, which is an important wavelength for application of detection of gases with hydrocarbons. To the best of our knowledge, this is the first demonstration of a free-standing polycrystalline $\mathrm{Si} \mathrm{PhC}$ membrane to be used in MIR region. High annealing temperature is used to reduce the residual stress in the membrane. In order to further reinforce the mechanical strength of the $\mathrm{PhC}$ membrane, $\mathrm{PhC}$ with square air holes was developed. Compared to circular air holes, $\mathrm{PhC}$ with square air holes maintains high reflectivity in MIR region. However, square air holes when compared to circular air holes, have a lower filling factor, which is defined as the volume of air holes over the volume of $\mathrm{Si}$ within a unit cell. This makes the membrane less brittle while keeping the reflectance around $3.45 \mu \mathrm{m}$ more than $90 \%$. To illustrate the feasibility of implementing such 2-D PhC mirrors in FPI, simulations based on the fabricated dimensions as well as optimized values are done. The filtered peak shows a full width half maximum (FWHM) of $0.08 \mathrm{~nm}$ which corresponds to a quality factor (Q-factor) of around 43800 .

\section{DESIGN, Modeling, AND FABrication}

The schematic of the design with air holes is shown in Fig. 1(a). The radius of the air hole is indicated as $\mathbf{r}$, the lattice constant is defined as a and the thickness of the $\mathrm{PhC}$ membrane is $\mathbf{t}$. In our proposed $\mathrm{PhC}$ device, the ratio of $\mathbf{r} / \mathbf{a}$ and $\mathbf{t} / \mathbf{a}$ are set to be $0.395(2 \pi \mathrm{c} / \mathrm{a})$ and $0.513(2 \pi \mathrm{c} / \mathrm{a})$ respectively.

Fig. 2 shows the corresponding band structure of the $\mathrm{PhC}$ design that was calculated based on plane wave expansion [36]. As can be seen, a band gap (shaded in red) is found from $0.528(2 \pi \mathrm{c} / \mathrm{a})$ to $0.587(2 \pi \mathrm{c} / \mathrm{a})$. In order to have a high reflectivity at $3.45 \mu \mathrm{m}$, the lattice constant is determined to be $1.92 \mu \mathrm{m}$. The radius is calculated to be $0.758 \mu \mathrm{m}$ and the thickness of the $\mathrm{Si}$

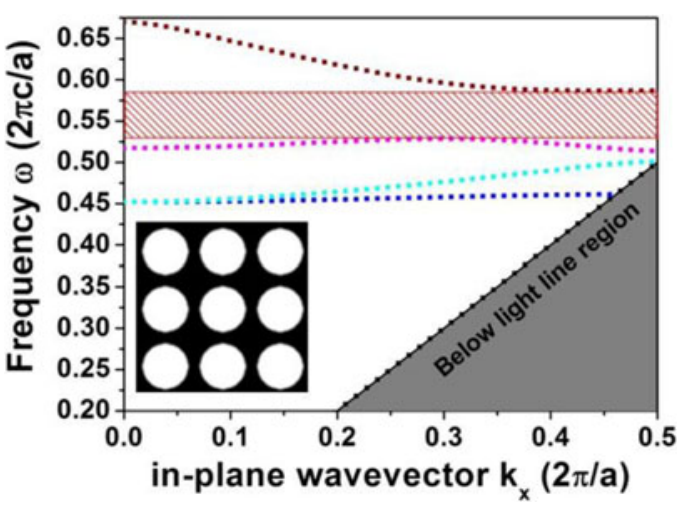

Fig. 2. Band structure of the propose $\mathrm{PhC}$ with circle air holes with the band gap region shaded in red.

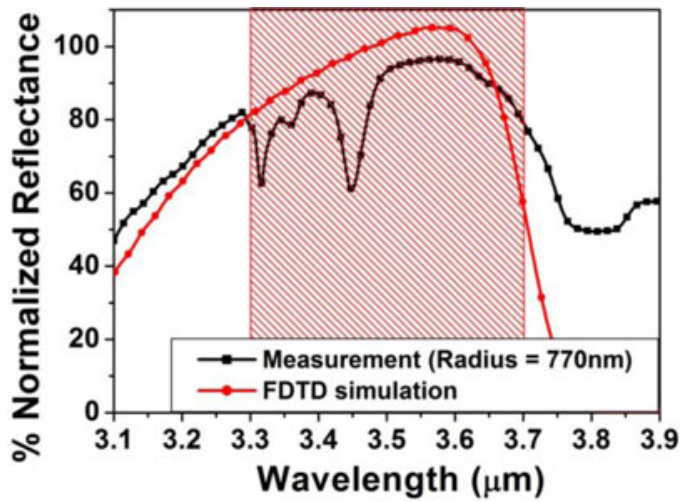

Fig. 3. Simulation of the reflectance based on the fabricated $\mathrm{PhC}$ with circle air holes overlaid with the measurement result.

membrane is $0.985 \mathrm{um}$. Fabrication of the $\mathrm{PhC}$ began with a bare Si wafer and a $1 \mu \mathrm{m}$ thick silicon oxide (BOX) was grown using thermal oxidation. This was followed by a $1 \mu \mathrm{m}$ thick PECVD Si layer which acts as the device layer. In order to ensure that the Si device layer is polycrystalline and to reduce the residual stress of the eventual suspended membrane, a high temperature anneal of $1000^{\circ} \mathrm{C}$ was done for $30 \mathrm{~min}$. The surface was then patterned by using deep-UV lithography and deep reactive ion etching (DRIE). In Fig. 1(b), the scanning electron microscope (SEM) photograph is shown. Due to fabrication uncertainties, the radius of the air holes is $0.77 \mu \mathrm{m}$ and the lattice constant is $1.95 \mu \mathrm{m}$. The thickness of the device layer is measured as $1 \mu \mathrm{m}$.

Simulation is done using finite difference time domain (FDTD) methodology to examine the performance of the $\mathrm{PhC}$ membranes. The refractive index of the $\mathrm{Si}$ is assumed to be 3.464 and the boundary conditions of the unit cell is set to periodic. As shown in Fig. 3, the simulated reflectivity displays a peak around $3.60 \mu \mathrm{m}$ and more than $90 \%$ reflectivity over a $286 \mathrm{~nm}$ range. In addition, it also matches well with the band gap region from the band structure calculated in Fig. 2, where the reflectance is high within the band gap region and experiences a drop once outside it. Generally, the simulated result agrees well with measurement result expect for two dips in reflectivity at $3.31 \mu \mathrm{m}$ and $3.45 \mu \mathrm{m}$ which will be accounted later in the paper. The higher reflectivity measured after $3.70 \mu \mathrm{m}$ wavelength is due to the reflection caused by the presence of the substrate. 

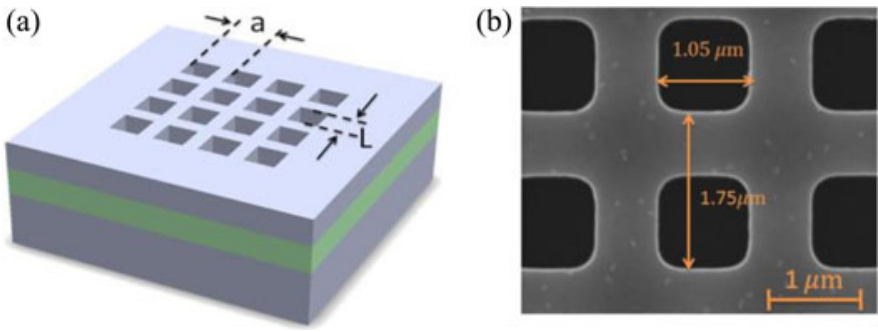

Fig. 4. (a) Schematic of the structure with square air holes and (b) SEM image of the fabricated device and (d) simulation of the reflectance.

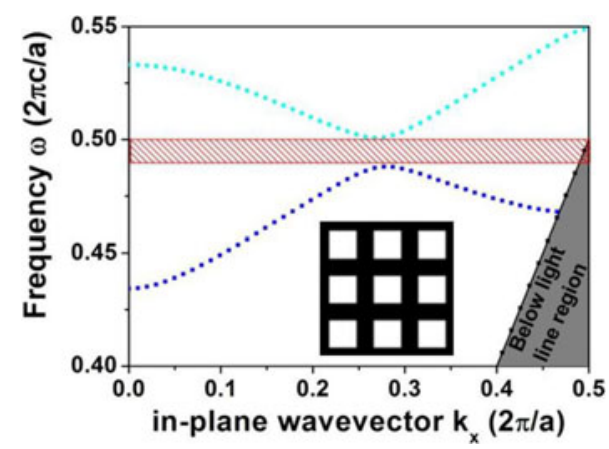

Fig. 5. Band structure of the propose $\mathrm{PhC}$ with square air holes with the band gap region shaded in red.

Similar approach is adopted for the design of the square air holes in Fig. 4(a). The length of the square air hole, $\mathbf{L}$, is designed to be $0.618 \mathbf{a}$ and the thickness, $\mathbf{t}$, is set to be $0.588 \mathbf{a}$. Based on the band gap region from $0.488(2 \pi \mathrm{c} / \mathrm{a})$ to $0.501(2 \pi \mathrm{c} / \mathrm{a})$ in Fig. 5, the lattice constant is determined to be $1.70 \mu \mathrm{m}$. This equates to an $\mathbf{L}$ of $1.05 \mu \mathrm{m}$ and $\mathbf{t}$ of $1 \mu \mathrm{m}$. The SEM photograph of the fabricated PhC membrane is shown in Fig. 4(b). Similar to the circle air holes, fabrication uncertainties caused the measured length of the square air holes to be $1.05 \mu \mathrm{m}$ and the lattice constant to be $1.75 \mu \mathrm{m}$. The device layer of the square air holes is still $1 \mu \mathrm{m}$. It can be observed that the square air holes are rounded and the radius of the curvature of the edges is estimated to be around $200 \mathrm{~nm}$. Based on FDTD simulation on the effect of rounded edges, this is within the tolerance that is allowed for a high reflectance. From the fabricated parameters, it can be calculated that the filling factor of the square air holes in the membrane is $36 \%$. In contrast, the filling factor for circular air holes is $49 \%$. The lower filling factor allows the membrane to be mechanical stronger and hence less brittle. This allows more flexibility in design and fabrication especially in MEMS application where such membranes are released and free-standing, as demonstrated in our $\mathrm{PhC}$ design. Simulation is also performed using FDTD calculation. The simulated result shows a high peak around $3.55 \mu \mathrm{m}$ in Fig. 6 and this match well with the measurement data.

\section{DEVICE ChARACTERIZATION}

The fabricated devices are characterized using Agilent Cary 620 FTIR Microscope where input wavelengths ranging from around $2 \mu \mathrm{m}$ to $8 \mu \mathrm{m}$ are incident perpendicularly onto the designed $\mathrm{PhC}$. The reflected light from the membrane is then

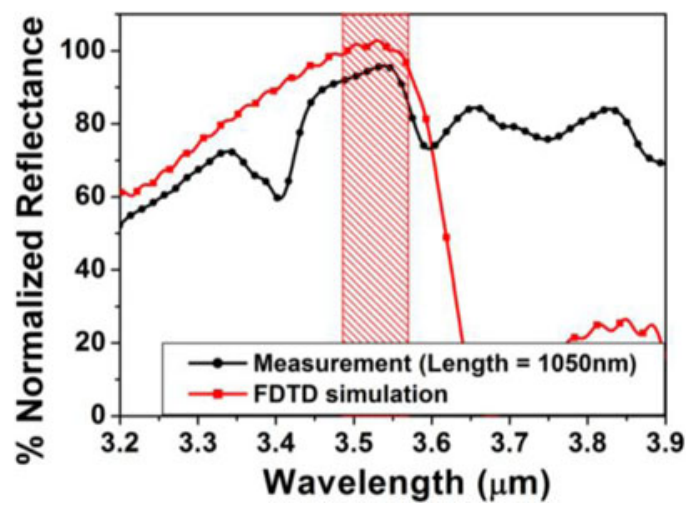

Fig. 6. Simulation of the reflectance based on the fabricated $\mathrm{PhC}$ with square air holes overlaid with the measurement result.

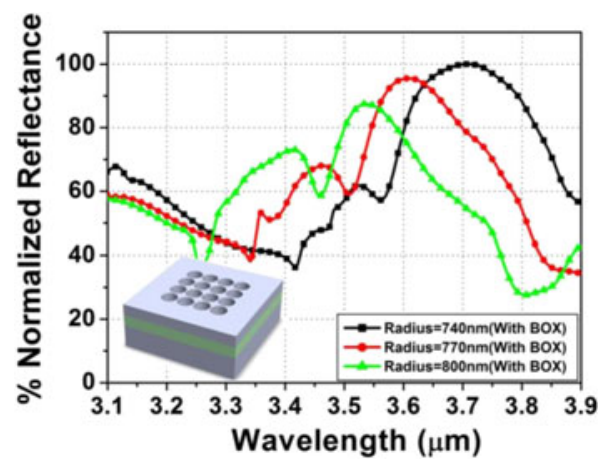

Fig. 7. Experimental results of stationary circle air holes design (with BOX).

collected by the mercury cadmium telluride detector. The measured reflectance is normalized against a gold sample which is assumed to have an almost perfect reflectance across the interested wavelengths [37]. The membrane size of the $\mathrm{PhC}$ is designed to be 300 by $300 \mu \mathrm{m}^{2}$ and the beam emitted by the FTIR microscope is 250 by $250 \mu \mathrm{m}^{2}$. This ensures that the illuminated beam is shone entirely on the patterned region in order to achieve maximum accuracy of the measurement. The experimental results of circle air holes are shown in Fig. 7. The measured reflectance of the circle air holes of radius $770 \mathrm{~nm}$ shows a peak of $95.5 \%$ at wavelengths around $3.61 \mu \mathrm{m}$ and reflectance greater than $90 \%$ spans from $3.56 \mu \mathrm{m}$ to $3.68 \mu \mathrm{m}$. As the radius of the circle air holes changes, it is observed that the reflected bandwidth experiences a blueshift as the radius increases. This can be attributed to the slight shift in the band gap region towards higher frequencies as the ratio of $\mathbf{r} / \mathbf{a}$ increases. Outside the band gap region, low reflectance values are measured. It is expected as the light enters one of the propagation modes sandwiching the band gap region.

For an increased performance of the $\mathrm{PhC}$ membrane with circle air holes, the BOX layer is removed by isotropic etching using vapour hydrofluoric acid (VHF). The schematic of the released structure is shown in the inset of Fig. 8. The measured reflectance of the $\mathrm{PhC}$ membrane with different radius of the circle air holes is shown as well. A peak of $96.5 \%$ reflectance is observed at $3.58 \mu \mathrm{m}$ for circular air holes with radius of $770 \mathrm{~nm}$. Generally, the spectra measured for the released PhC 


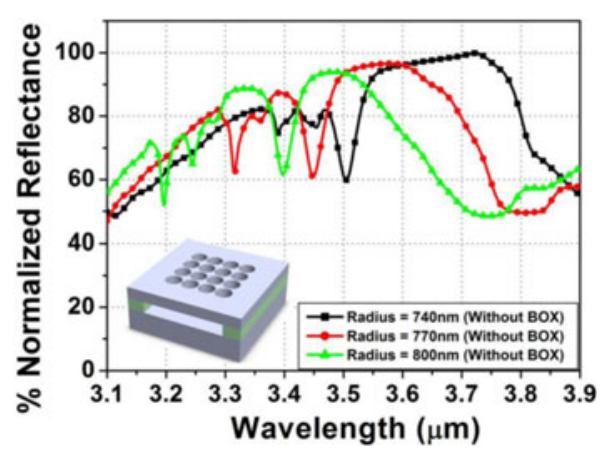

Fig. 8. Experimental results of released cicle air holes design (without BOX).

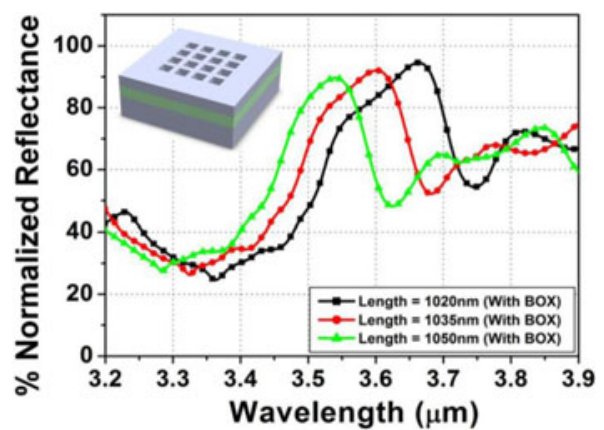

Fig. 9. Experimental results of stationary square air holes design (with BOX).

membrane with circle air holes shows a distinct blueshift when compared to the unreleased circle air holes design. As the BOX layer is removed, the refractive index of the cladding below the $\mathrm{PhC}$ membrane decreased from 1.44 (silicon oxide) to 1 (air). This reduces the effective refractive index which leads to the movement of the spectra towards lower wavelengths. Two reflectivity dips are also observed at $3.31 \mu \mathrm{m}$ and $3.45 \mu \mathrm{m}$. As mentioned, reflection from the Si substrate causes increase in reflectance. However, destructive interference occurs at these two wavelengths and this causes the reflectance to display dips in the spectrum.

Fig. 9 shows the experimental results of 300 by $300 \mu \mathrm{m}^{2}$ unreleased $\mathrm{PhC}$ membrane with square air holes. For square air holes with length of $1035 \mathrm{~nm}$, a reflectance peak of $92.0 \%$ is present at $3.60 \mu \mathrm{m}$ wavelength. The high reflectance region spans across a much smaller bandwidth from $3.58 \mu \mathrm{m}$ to $3.61 \mu \mathrm{m}$. Similar to the $\mathrm{PhC}$ membrane with circle air holes, when the length of the square air holes increases, the reflectance peak shows blueshift because more area within the membrane becomes air which has a lower refractive index. This causes the band gap region moving towards higher frequencies. After the BOX layer is removed using isotropic etching by VHF, the reflectance is enhanced as shown in Fig. 10. The measured reflectance for the square air holes with length $1035 \mathrm{~nm}$ is $97.2 \%$ at $3.59 \mu \mathrm{m}$ wavelength. Similar blueshift can be seen when the length of the square air holes is increased.

In summary, the measurement results of both $\mathrm{PhC}$ with circular air holes and square air holes verify that $\mathrm{PhC}$ with square air holes shows high reflectivity that is comparable to $\mathrm{PhC}$ with circular air holes. While not sacrificing optical performance,

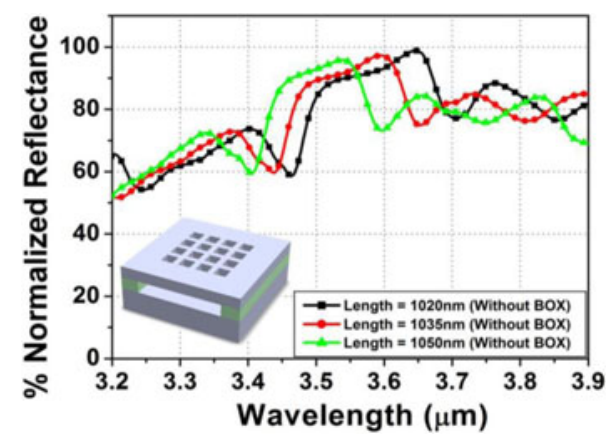

Fig. 10. Experimental results of released square air holes design (without BOX). (a)

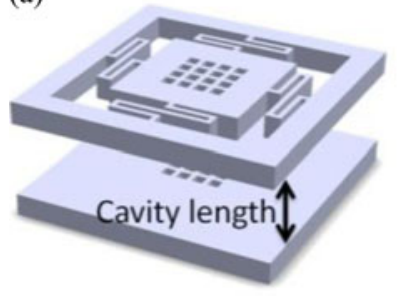

(b)

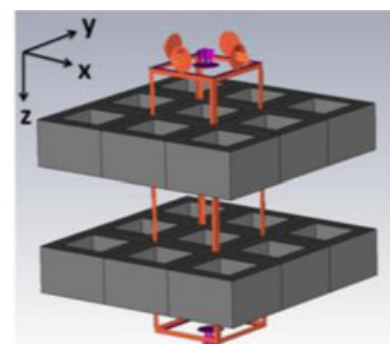

Fig. 11. (a) Schematic of FPI using PhCs as mirrors and (b) model of PhC membrane used in simulation programme, CST MWS.

square air holes offers better mechanical strength due to its lower filling factor of $36 \%$. In contrast, circular air holes are larger in size and hence have a higher filling factor of $49 \%$. This makes square air holes a more attractive candidate especially in MEMS applications.

\section{Simulation OF FPI}

In this section, the feasibility of implementing 2-D PhC designs as high reflectivity mirror in FPI is explored using simulations done by computer simulation technology microwave studio (CST MWS). As discussed previously, such high reflectivity mirrors are commonly implemented using multi-layer structures which faces the problem of high residual stress in the layers. In contrast, 2-D PhC designs are able to achieve high reflectivity using a thin $\mathrm{Si}$ membrane which is light weight and easy to fabricate.

The schematic of the FPI is shown in Fig. 11(a). The PhC membranes are used as the mirrors and the top Si PhC membrane is supported using springs. The cavity length can be made tunable by using MEMS techniques like applying a voltage difference between the top and bottom Si slab. This induces an attractive electrostatic force which will pull the top and bottom slabs towards each other, hence reducing the gap between them. The transmission output of the FPI as the cavity length changes is simulated using CST MWS. The simulation model is shown in Fig. 11(b). Only the unit cell indicated by the red box is simulated and periodic boundary condition is set on all four sides. This helps to reduce computation time while maintaining high accuracy in the simulated output. In order to show the feasibility of using square air holes to be used in FPI, we will compare its performance with that of circular air holes. 


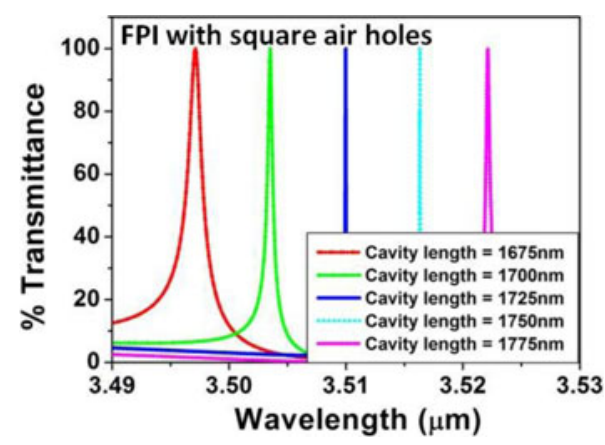

Fig. 12. Simulated transmittance of the FPI with various cavity length using square air holes.

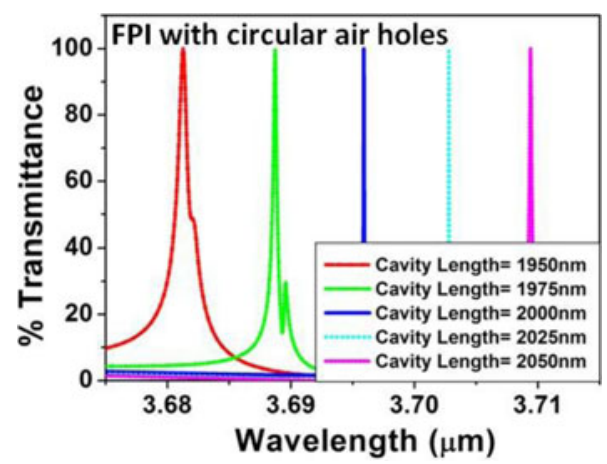

Fig. 13. Simulated transmittance of the FPI with various cavity length using circle air holes.

In Fig. 12, the simulated spectrum of the FPI using square air holes is presented. In the simulations, the inputted light is propagating along the $\mathrm{z}$ direction and the electric field is along the $\mathrm{x}$ direction. The values of $\mathbf{L}, \mathbf{a}$ and $\mathbf{t}$ are $1.05 \mu \mathrm{m}$, $1.70 \mu \mathrm{m}$ and $1 \mu \mathrm{m}$ respectively. The FPI shows a tuning range of $25 \mathrm{~nm}$ from $3.497 \mu \mathrm{m}$ to $3.522 \mu \mathrm{m}$ when the cavity length is changed $1.675 \mu \mathrm{m}$ to $1.775 \mu \mathrm{m}$. When the cavity length is $1.725 \mu \mathrm{m}$, the FWHM of the simulated peak is $0.08 \mathrm{~nm}$ which corresponds to a Q-factor of 43800 . This is around an order of magnitudes higher than the simulated Q-factor of FPI using multi-layered structures [29], [32], [34]. The enhanced Q-factor is attributed to the dual filtering effect of the $\mathrm{PhC}$ membrane. The first filtering effect is due to the destructive interference of the undesired wavelength within the Fabry-Perot cavity. The second filtering effect is due to the wavelength selective reflectivity that is intrinsic in the PhC design. In order to fully assess the practicability of using square air holes in FPI, FPI with circular air holes mirrors are also simulated for comparison using similar method mentioned above. The radius of the circular air hole is $770 \mathrm{~nm}$ and the lattice constant is $1.95 \mu \mathrm{m}$. The top and bottom Si slabs are maintained at $1 \mu \mathrm{m}$. The simulated results are presented in Fig. 13. The FWHM of the transmittance peak corresponding to a cavity length of $2 \mu \mathrm{m}$ is $0.07 \mathrm{~nm}$ which equates to a Q-factor of about 52000. The tuning range of the FPI is $28 \mathrm{~nm}$ from $3.681 \mu \mathrm{m}$ to $3.709 \mu \mathrm{m}$ when the cavity length is changed $1.950 \mu \mathrm{m}$ to $2.050 \mu \mathrm{m}$.

Based on the simulations of the FPI using both circle air holes and square air holes, it has been shown the proposed
$\mathrm{PhC}$ designs are ideal candidates for the realization of the high reflectivity mirrors. At the same time, the use of $\mathrm{PhC}$ with square air holes in FPI also displays good optical performance which is comparable to $\mathrm{PhC}$ with circle air holes. As described in pervious section, using $\mathrm{PhC}$ with square air holes offers higher mechanical strength which is vital in applications such as FPI where the PhC mirrors are free-standing.

\section{CONCLUSION}

In conclusion, two designs of 2-D PhC based polycrystalline $\mathrm{Si}$ membranes have been demonstrated as ultra-compact $\mathrm{Si}$ based reflector. Both the circle air holes and square air holes designs were measured to show $96.5 \%$ and $97.2 \%$ reflectance around $3.58 \mu \mathrm{m}$, which is an important wavelength for hydrocarbon gas sensing in the MIR region. While not sacrificing optical performance, $\mathrm{PhC}$ with square air holes offers more mechanical strength due to its lower filling factor of $36 \%$. The feasibility of implementing such 2-D PhC design as mirrors in FPI was also explored. It was shown through simulations that by using $\mathrm{PhC}$ with square air holes as mirrors in the FPI, the output peak has a FWHM of $0.08 \mathrm{~nm}$ and a Q-factor of around 43800. This demonstrates clearly the potential of using such 2-D PhC mirrors to form compact FPI for high resolution applications such as gas sensing and hyperspectral imaging.

\section{REFERENCES}

[1] L. Hui, Y. Lianshan, G. Zhen, P. Wei, W. Kunhua, L. Hengyi, and X. Luo "Enhanced focusing properties using surface plasmon multilayer gratings," IEEE Photon. J., vol. 4, no. 1, pp. 57-64, Feb. 2012.

[2] M. Carras, G. Maisons, B. Simozrag, M. Garcia, O. Parillaud, J. Massies, and X. Marcadet, "Room-temperature continuous-wave metal grating distributed feedback quantum cascade lasers," Appl. Phys. Lett., vol. 96, no. 16, pp. 161105-1-161105-3, Apr. 2010.

[3] L. Liyang, L. Fei, X. Mu, W. Tao, W. Jiayang, Z. Linjie, and S. Yikai, "Mode-selective hybrid plasmonic bragg grating reflector," IEEE Photon. Technol. Lett., vol. 24, no. 19, pp. 1765-1767, Oct. 2012.

[4] Z. Yu, P. Deshpande, W. Wei, J. Wang, and S. Y. Chou, "Reflective polarizer based on a stacked double-layer subwavelength metal grating structure fabricated using nanoimprint lithography," Appl. Phys. Lett., vol. 77, no. 7, pp. 927-929, Aug. 2000.

[5] A. Liu, F. Fu, Y. Wang, B. Jiang, and W. Zheng, "Polarization-insensitive subwavelength grating reflector based on a semiconductor-insulator-metal structure," Opt. Exp., vol. 20, no. 14, pp. 14991-15000, 2012.

[6] D. A. Horsley, J. Provine, and J. Skinner, "Integration of subwavelength metal gratings with MEMS actuators," Proc. SPIE, vol. 6008, pp. 600810 600811, Nov. 2005.

[7] C. Ndiaye, F. Lemarchand, M. Zerrad, D. Ausserré, and C. Amra, "Optimal design for $100 \%$ absorption and maximum field enhancement in thin-film multilayers at resonances under total reflection," Appl. Opt., vol. 50, no. 9, pp. C382-C387, 2011.

[8] T. M. Jordan, J. C. Partridge, and N. W. Roberts, "Non-polarizing broadband multilayer reflectors in fish," Nat. Photon., vol. 6, pp. 759-763, Oct. 2012.

[9] T. D. Corrigan, D. H. Park, H. D. Drew, S.-H. Guo, P. W. Kolb, W. N. Herman, and R. J. Phaneuf, "Broadband and mid-infrared absorber based on dielectric-thin metal film multilayers," Appl. Opt., vol. 51, no. 8, pp. 1109-1114, 2012.

[10] W. Shen, X. Sun, Y. Zhang, Z. Luo, X. Liu, and P. Gu, "Narrow band filters in both transmission and reflection with metal/dielectric thin films," Opt. Commun., vol. 282, no. 2, pp. 242-246, Jan. 2009.

[11] S. Yichen, Z. Deyin, G. Medhi, R. Peale, M. Zhenqiang, W. Buchwald, R. Soref, and Z. Weidong, "Fano-resonance photonic crystal membrane reflectors at mid- and far-infrared," IEEE Photon. J., vol. 5, no. 1, p. 4700206 Feb. 2013. 
[12] Q. Zexuan, Y. Hongjun, S. Chuwongin, Z. Deyin, M. Zhenqiang, and Z. Weidong, "Design of fano broadband reflectors on SOI," IEEE Photon. Technol. Lett., vol. 22, no. 15, pp. 1108-1110, Aug. 2010.

[13] S. Fan and J. D. Joannopoulos, "Analysis of guided resonances in photonic crystal slabs," Phys. Rev. B, vol. 65, no. 23, pp. 235112-1-235112-8, 2002

[14] J. Song, R. Proietti Zaccaria, M. B. Yu, and X. W. Sun, "Tunable Fano resonance in photonic crystal slabs," Opt. Exp., vol. 14, no. 19, pp. 88128826, Sep. 2006.

[15] J. Il Woong, S. Basu Mallick, and O. Solgaard, "A large-area highreflectivity broadband monolithic single-crystal-silicon photonic crystal mirror MEMS scanner with low dependence on incident angle and polarization," IEEE J. Sel. Top. Quantum Electron., vol. 15, no. 5, pp. 14471454, Sep. 2009.

[16] Y. Zhang, M. Khan, Y. Huang, J. Ryou, P. Deotare, R. Dupuis, and M. Loncar, "Photonic crystal nanobeam lasers," Appl. Phys. Lett., vol. 97, no. 5, pp. 051104-1-051104-3, Aug. 2010.

[17] H. Yang, D. Zhao, S. Chuwongin, J.-H. Seo, W. Yang, Y. Shuai, J. Berggren, M. Hammar, Z. Ma, and W. Zhou, "Transfer-printed stacked nanomembrane lasers on silicon," Nat. Photon., vol. 6, no. 9, pp. 615-620, Jul. 2012.

[18] Y. Wang, Y. Kanamori, H. Zhu, and K. Hane, "Single layer silicon photonic crystal slab," Photon. Nanostruct.—Fundam. Appl., vol. 10, no. 1, pp. 146 152, Jan. 2012.

[19] K. B. Crozier, V. Lousse, O. Kilic, S. Kim, S. Fan, and O. Solgaard, "Airbridged photonic crystal slabs at visible and near-infrared wavelengths," Phys. Rev. B, vol. 73, no. 11, pp. 115126-1-115126-14, 2006.

[20] S. Basu Mallick, J. Il Woong, A. M. Meisner, J. Provine, R. T. Howe, and O. Solgaard, "Multilayered monolithic silicon photonic crystals," IEEE Photon. Technol. Lett., vol. 23, no. 11, pp. 730-732, Jun. 2011.

[21] R. Shankar, R. Leijssen, I. Bulu, and M. Loncar, "Mid-infrared photonic crystal cavities in silicon," Opt. Exp., vol. 19, no. 6, pp. 5579-5586, 2011.

[22] S. Y. Lin, J. G. Fleming, D. L. Hetherington, B. K. Smith, R. Biswas, K. M. Ho, M. M. Sigalas, W. Zubrzycki, S. R. Kurtz, and J. Bur, "A threedimensional photonic crystal operating at infrared wavelengths," Nature, vol. 394, no. 6690, pp. 251-253, Apr. 1998.

[23] M. Muneeb, X. Chen, P. Verheyen, G. Lepage, S. Pathak, E. Ryckeboer A. Malik, B. Kuyken, M. Nedeljkovic, J. Van Campenhout, G. Z. Mashanovich, and G. Roelkens, "Demonstration of Silicon-oninsulator mid-infrared spectrometers operating at $3.8 \mu \mathrm{m}$," Opt. Exp., vol. 21 , no. 10 , pp. 11659-11669, 2013.

[24] C. Reimer, M. Nedeljkovic, D. J. M. Stothard, M. O. S. Esnault, C. Reardon, L. O'Faolain, M. Dunn, G. Z. Mashanovich, and T. F. Krauss, "Mid-infrared photonic crystal waveguides in silicon," Opt. Exp., vol. 20, no. 28, pp. 29361-29368, 2012.

[25] A. Torkkeli, O. Rusanen, J. Saarilahti, H. Seppä, H. Sipola, and J. Hietanen, "Capacitive microphone with low-stress polysilicon membrane and highstress polysilicon backplate," Sens. Actuators A, Phys., vol. 85, no. 1-3, pp. 116-123, Aug. 2000.

[26] Y. Jie, H. Kahn, H. An-Qiang, S. M. Phillips, and A. H. Heuer, "A new technique for producing large-area as-deposited zero-stress LPCVD polysilicon films: The multipoly process," J. Micro-Electromech. Syst., vol. 9, no. 4, pp. 485-494, Dec. 2000.

[27] S. Kim, S. Hadzialic, A. S. Sudbo, and O. Solgaard, "Reflectivity and polarization dependence of polysilicon single-film broadband photonic crystal micro-mirrors," Opt. Exp., vol. 20, no. 6, pp. 6306-6315, 2012.

[28] J. Wöllenstein, A. Eberhardt, S. Rademacher, and K. Schmitt, "Miniaturized multi channel infrared optical gas sensor system," Proc. SPIE, vol. 8066, pp. 80660Q-1-80660Q-8, May 2011.

[29] J. Mayrwöger, C. Mitterer, W. Reichl, C. Krutzler, and B. Jakoby, "Fabryperot-based thin film structure used as IR-emitter of an NDIR gas sensor: Ray tracing simulations and measurements," Proc. SPIE, vol. 8066, pp. 80660k-1-80660k-10, May 2011.

[30] A. J. Keating, J. Antoszewski, K. K. M. B. D. Silva, K. J. Winchester, T. Nguyen, J. M. Dell, C. A. Musca, L. Faraone, P. Mitra, J. D. Beck, M. R. Skokan, and J. E. Robinson, "Design and characterization of fabrypérot MEMS-based short-wave infrared microspectrometers," J. Electron. Mater, vol. 37, no. 12, pp. 1811-1820, Dec. 2008.

[31] C. A. Musca, J. Antoszewski, K. J. Winchester, A. J. Keating, T. Nguyen, K. K. M. B. D. Silva, J. M. Dell, L. Faraone, P. Mitra, J. D. Beck, M. R. Skokan, and J. E. Robinson, "Monolithic integration of an infrared photon detector with a MEMS-based tunable filter," IEEE Electron Dev. Lett., vol. 26, no. 12, pp. 888-890, Dec. 2005.

[32] J. S. Milne, J. M. Dell, A. J. Keating, and L. Faraone, "Widely tunable MEMS-based Fabry Perot filter," J. Microelectromech. Syst., vol. 18, no. 4 pp. 905-913, Aug. 2009.
[33] T. J. Russin, M. Kerber, A. Russin, A. Wang, and R. Waters, "Fabrication and analysis of a MEMS NIR Fabry Perot interferometer," J. Microelectromech. Syst., vol. 21, no. 1, pp. 181-189, Feb. 2012.

[34] M. Malak, F. Marty, N. Pavy, Y. A. Peter, L. Ai-Qun, and T. Bourouina, "Cylindrical surfaces enable wavelength-selective extinction and Sub$0.2 \mathrm{~nm}$ linewidth in $250 \mu \mathrm{m}$-gap silicon Fabry-Perot cavities," J. Microelectromech. Syst., vol. 21, no. 1, pp. 171-180, Feb. 2012.

[35] W. Suh, M. F. Yanik, O. Solgaard, and F. Shanhui, "Displacement-sensitive photonic crystal structures based on guided resonance in photonic crystal slabs," Appl. Phys. Lett., vol. 82, no. 13, pp. 1999-2001, Mar. 2003.

[36] S. Johnson and J. Joannopoulos, "Block-iterative frequency-domain methods for Maxwell's equations in a planewave basis," Opt. Exp., vol. 8, no. 3 , pp. 173-190, Jan. 2001.

[37] D. B. Nash, "Mid-infrared reflectance spectra $(2.3-22 \mu \mathrm{m})$ of sulfur, gold, KBr, MgO, and halon," Appl. Opt., vol. 25, no. 14, pp. 2427-2433, 1986.

Chong Pei Ho received the B.Eng. degree from the National University of Singapore, Singapore, in 2011, where he is currently a Research Engineer and has been working toward the Ph.D. degree since January 2012. His research interests include applications involving nanophotonics.

Prakash Pitchappa received the B.Eng. degree in electronics and communications from Anna University, Chennai, India, in 2008 and the M.Sc. degree from the National University of Singapore (NUS), Singapore, where he has been working toward the Ph.D. degree since January 2012. He is currently a Research Engineer at NUS and is attached to the Institute of Microelectronics, Agency for Science, Technology and Research, Singapore. His research interests include development and fabrication MEMS-based metamaterials devices.

Piotr Kropelnicki was born in Leszno, Poland, on 20 September 1981. He received the Diplom Ingenieur degree in electrical and electronics engineering from Universität Duisburg-Essen, Germany, in 2007, with major on microelectronics. He received the Ph.D. degree in microelectronics at the Fraunhofer Institute for Microelectronic Circuit and Systems, Fraunhofer, Germany, in 2010. $\mathrm{He}$ is currently a Principal Investigator, leading a team in SAM-Sensors \& Actuators Microsystems Program at the Institute of Microelectronics, Agency for Science, Technology and Research, Singapore. He is responsible for the development of several sensors operating in harsh environment, like pressure, gas detection, optical, viscosity, and temperature sensors.

Jian Wang received the B.Eng. degree from the National University of Singapore, Singapore. He is currently in the Department of MEMS of the Institute of Microelectronics, Agency for Science, Technology and Research, Singapore. His research interest includes MEMS integration processes.

Yandong Gu received the Ph.D. and M.E.E. degrees from the University of Minnesota, Minneapolis, MN, USA, in 2002. He is the Technical Director of the Institute of Microelectronics, Agency for Science, Technology and Research, Singapore. His research interests include optical-based chemical sensing, AlNbased sensors and actuators, and miniaturized medical instrumentation. 
Chengkuo Lee (S'93-M'96) received the M.S. degree in materials science and engineering from National Tsing Hua University, Hsinchu, Taiwan, in 1991, the M.S. degree in industrial and system engineering from Rutgers University, New Brunswick, NJ, USA, in 1993, and the Ph.D. degree in precision engineering from the University of Tokyo, Tokyo, Japan, in 1996.

He was a Foreign Researcher in the Nanometerscale Manufacturing Science Laboratory at the Research Center for Advanced Science and Technology, University of Tokyo from 1993 to 1996 . He had also worked in the Technical Engineering Laboratory, Advanced Industrial Science and Technology, Ministry of International Trade and Industry of Japan, as a Japan Science and Technology Research Fellow, in 1996. Thereafter, he was a Senior Research Staff Member of the Microsystems Laboratory, Industrial Technology Research Institute, Hsinchu. In September 1997, he joined the Metrodyne Microsystem Corporation, Hsinchu, and established the MEMS Device Division and the first micromachining laboratory for commercial purposes in Taiwan.

He was the Manager of the MEMS Device Division between 1997 and 2000. He was an Adjunct Assistant Professor in the Department of Electrophysics, National Chiao Tung University, Hsinchu in 1998, and an Adjunct Assistant Professor in the Institute of Precision Engineering, National Chung Hsing University, Taichung, Taiwan, from 2001 to 2005. He cofounded Asia Pacific Microsystems, Inc. (APM), Hsinchu, in August 2001, and he became the Vice President of R\&D; then, later, until the end of 2005, he became the Vice President of the optical communication business unit and the Special Assistant to the Chief Executive Officer in charge of international business and technical marketing for MEMS foundry service at APM, Inc., one of the top 30 MEMS manufacturers in the world in 2004. From 2006 to 2009, he was a Senior Member of Technical Staff at the Institute of Microelectronics, Agency for Science, Technology and Research, Singapore. He is currently an Associate Professor in the Department of Electrical and Computer Engineering, National University of Singapore, Singapore. He is the coauthor of the book Advanced MEMS Packaging (McGraw-Hill, 2010). He has contributed more than 190 international Conference papers and extended abstracts, 135 peer-reviewed international journal articles, and nine U.S. patents in the MEMS, NEMS, Metamaterials, nanophotonics, and nanotechnology fields. 